\title{
Naloxone Counteracts the Promoting Tumor Growth Effects Induced by Morphine in an Animal Model of Triple-negative Breast Cancer
}

\author{
SABRINA BIMONTE ${ }^{1 *}$, ANTONIO BARBIERI $^{2 *}$, MARCO CASCELLA $^{1 *}$, DOMENICA REA $^{2}$, GIUSEPPE PALMA $^{2}$, \\ ANTONIO LUCIANO ${ }^{2}$, CIRA ANTONIETTA FORTE ${ }^{1}$, ARTURO CUOMO $^{1 \#}$ and CLAUDIO ARRA ${ }^{2 \#}$ \\ ${ }^{1}$ Division of Anesthesia and Pain Medicine, Istituto Nazionale Tumori, \\ IRCCS Fondazione G. Pascale, Naples, Italy; \\ ${ }^{2}$ S.S.D. Sperimentazione Animale, Istituto Nazionale Tumori, IRCCS Fondazione G. Pascale, Naples, Italy
}

\begin{abstract}
Background/Aim: Our group has previously demonstrated, in in vitro and in vivo studies on triple-negative breast cancer, that morphine promoted breast cancer progression whereas naloxone was able to reduce it. In this subsequent investigation, we aimed to assess the combinatorial effects of these two drugs in an animal model of triple negative breast cancer. Materials and Methods: In order to evaluate the in vivo effects of the combination of morphine and naloxone in human breast cancer, a mouse model of human triple-negative breast cancer was generated by injecting the MDA-MB-231 cells subcutaneously in nude mice. Naloxone and morphine were daily intraperitoneally coinjected in mice for 4 weeks at two different doses. Microvessel formation was detected by fluorescein isothiocyanatedextran $(100 \mu l)$ injected into the lateral tail vein of mice and confirmed by immunohistochemistry for PECAM-1 on mice tumor sections. Results: In vivo experiments showed that naloxone was able to counteract the promoting effects of morphine on tumor growth. No impairment of micro-vessel formation in tumors of mice treated with the two drugs was observed. Conclusion: Herein, we demonstrated that naloxone was able to counteract the promoting effects of morphine on tumor growth without impairing micro-vessel formation.
\end{abstract}

This article is freely accessible online.

*These Authors contributed equally as first Authors.

\#These Authors contributed equally as last Authors.

Correspondence to: Sabrina Bimonte, Division of Anesthesia and Pain Medicine, Istituto Nazionale Tumori, IRCCS Fondazione G. Pascale, Via Mariano Semmola 53, 80131 Naples, Italy. Tel: +39 0815903221, e-mail: s.bimonte@istitutotumori.na.it

Key Words: Morphine, Naloxone, breast cancer, proliferation, angiogenesis.
Morphine is an opioid commonly used for pain management in cancer patients $(1,2)$. This drug acts directly on the central nervous system (CNS), through the brain opioid receptors, $\mu, \delta$, and $\mathrm{K}(3,4)$. Despite its significance in managing pain in cancer patients, pre-clinical studies have demonstrated that morphine acts as an inhibitor (5-7), or stimulator (8-10) of breast cancer cell growth and angiogenesis, depending on the doses used (11). Particularly, our group has previously demonstrated, in pre-clinical studies on triple-negative breast cancer, that morphine promoted angiogenesis and breast cancer progression (10). Interestingly, differently, from morphine, several reports have shed light on the inhibitory role of naloxone, the antagonist of morphine, in the proliferation of cancer cells (12-13). Specifically, we recently demonstrated that its antagonist, naloxone, inhibited the proliferation and enhanced the apoptosis of MDA-MB-231 cells in vitro, and inhibited tumor growth without affecting angiogenesis in vivo (14). This study aimed to dissect the effects of the combinations of these two drugs in an animal model of human breast cancer. Our results demonstrated that naloxone was able to counteract the promoting effects of morphine on tumor growth. No impairment in micro-vessel formation in tumors of treated mice was observed.

\section{Materials and Methods}

Drugs and reagents. Morphine hydrochloride employed was kindly provided by Dr. Arturo Cuomo (IRCCS Fondazione Pascale) and was dissolved in distilled water at $100 \mathrm{mM}$ concentration. Naloxone hydrochloride was obtained from (Molteni, Scandicci, Italy). The two drugs were used for in vitro tests in three different concentrations $(1,10,100 \mu \mathrm{M})$ on MDA-MB-231 cell line. The anti-p53 antibody was purchased by Imgenex (San Diego, CA, USA). The antibody against CD31 was provided by DAKO (Santa Cruz, CA, USA). 3, 3-diaminobenzidine horseradish peroxidase (HRP) substrate was purchased from Vector Laboratories (USA). Dulbecco's Modified Eagle's medium (DMEM), fetal bovine serum 
(FBS), penicillin, and streptomycin were obtained from Invitrogen (Grand Island, NY, USA). Bovine serum albumin (BSA) NaCl, Tris, sodium dodecyl sulfate (SDS) and glycine, were all from Sigma Chemical (St. Louis, MO, USA).

Cell culture. The human ER-negative breast cancer cell line MDAMB-231 was obtained from the American Type Culture Collection (ATCC ${ }^{\circledR}$ HTB-26 ${ }^{\mathrm{TM}}$ Manassas, VA, USA).

Xenograft breast cancer mouse model. Eight-week-old female Fox 1 nu/nu mice were purchased by Harlan, San Pietro al Natisone, Italy. Mice were housed in a group of five in a $12 \mathrm{~h}$ light: $12 \mathrm{~h}$ dark cycle in a controlled room temperature of $22 \pm 2^{\circ} \mathrm{C}$ and fed ad libitum. Before the injection of cancer cells, mice were anesthetized with a solution of Zoletil 100 (Virbac), injected intraperitoneally according to their weight. Xenograft mouse model of breast cancer was generated by injected MDA-MB-231 breast cancer cells $\left(2.5 \times 10^{6}\right)$ subcutaneously into the right flank in each mouse as we have previously described $(9,13)$. At the time of randomization when the tumor reached a volume of $\sim 30-60 \mathrm{~mm}^{3}$, mice $(\mathrm{n}=8)$ were equally divided into four groups on the basis of different treatments:

(a) Control group: four weeks of treatment with normal saline solution;

(b) Morphine group: four weeks of treatment $(0.714 \mathrm{mg} / \mathrm{kg}$ daily for 2 weeks and $1.43 \mathrm{mg} / \mathrm{kg}$ daily for the next 2 weeks);

(c) Naloxone group: four weeks of treatment weeks $(0.357 \mathrm{mg} / \mathrm{kg}$ daily for 2 weeks and $0.714 \mathrm{mg} / \mathrm{kg}$ daily for the next 2 weeks);

(d) Morphine plus naloxone group: 4 weeks of treatment $(0.714 \mathrm{mg} / \mathrm{kg}$ morphine plus $0.357 \mathrm{mg} / \mathrm{kg}$ daily for the first 2 weeks; $1.43 \mathrm{mg} / \mathrm{kg}$ morphine plus $0.714 \mathrm{mg} / \mathrm{kg}$ naloxone daily for the next 2 weeks). A digital caliper was used to measure tumor volume once a week and then we employed the following formula in order to estimate the tumor growth: volume $\left(\mathrm{mm}^{3}\right)=$ length $\times$ width $\times$ width $/ 2$. Paired $t$-test one-tailed analysis was used to examine the significance of difference among the four groups (Graph Pad Prism 5.0).

Macrofluo microscope was used to detect micro-vessel formation after the injection of FITC-dextran $(100 \mu \mathrm{l} /$ mouse $)$ in the lateral tail vein.

The study was approved by the Italian Animal Ethics Committee of Istituto Nazionale dei Tumori Fondazione G. Pascale of Naples, Italy. All experiments were performed by also following the European Directive 63/2010/UE and the Italian Law (DL 116/92, authorized by Minister of Health, Italy; Prot.N. 905/2013). This study was carried out in accordance with the recommendations that cover all scientific procedures involving the use of live animals as we have previously reported $(9,13)$.

Immunohistochemistry. After tumor ex vivo excision the samples from treated and control animals were embedded in paraffin and fixed in paraformaldehyde. After several PBS washings, the tumor was cut in slices and blocked for 20 min with protein block solution (DakoCytomation, Glostrup, Denmark) and then incubated overnight with polyclonal anti-goat PECAM-1 (1:100). The day after, the slices were washed and incubated with biotinylated link universal antiserum followed by horseradish peroxidase -streptavidin conjugate (LSAB+kit). The slides were rinsed, and the color was developed using 3, 3'-diaminobenzidine hydrochloride as a chromogen. In the end, the slices were flushed in distilled water, counterstained with hematoxylin, and coverslips were mounted on with DPX mounting medium. Photometric Cool

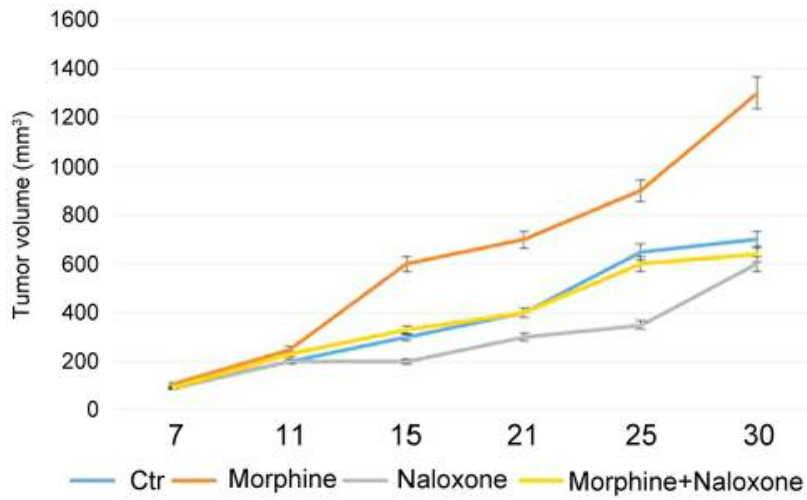

Figure 1. The effects of morphine and naloxone on tumor growth in a triple-negative breast cancer mouse model. Tumors were treated with vehicle, morphine alone, naloxone alone and morphine plus naloxone. Tumor volumes decreased after 21 days of treatment with two drugs until 30 days compared to controls and to mice treated with morphine alone.

SNAP CF color camera (Nikon, Lewisville, TX, USA) was used to take pictures that were analyzed with Image-ProPlus software (Version 6.0, Media Cybernetics, Bethesda, MD, USA). Digital images from three different section of the tumor were scan. Microvessel density (MVD) was counted according to previously described protocol $(7,14)$.

Statistical analyses. Statistical analysis was done with the support of SPSS version 18.0 (SPSS, Chicago, IL, USA). All experiments were performed in triplicate. Data were presented as mean \pm SD. Two-tailed $t$-test and $\mathrm{X}^{2}$ test were used for comparison between the different groups. $p<0.05$ was considered statistically significant.

\section{Results}

The effects of morphine and naloxone on tumor growth of triple-negative breast cancer. In order to dissect the effects of the combination of morphine and naloxone on tumor growth, we used a xenograft mouse model of TNBC. Our data showed that naloxone was able to counteract the promoting effects of morphine on breast tumor growth. Compared to controls and to mice treated with morphine alone, treatment of mice with both morphine and naloxone for 30 days resulted in inhibition of the growth of tumor (Figure 1). As we have previously reported (13), compared to controls, treatment of mice with naloxone for 35 days inhibited tumor growth, while morphine enhanced tumor growth (9).

Measurements of mice body weight did not show differences between the two groups of animals, thus suggesting minimal toxicity induced by two drugs (data not shown).

The effects of morphine and naloxone on angiogenesis in breast tumors. In our previous report, we have shown that 




Figure 2. Naloxone does not counteract the promoting effect of morphine on micro-vessel formation in breast tumors. (A-D) Measurements of fluorescence per sec depicting micro-vessel formation in breast tumors (Fitc-dextran) using MacroFluo microscope. Images show that naloxone $(C)$ does not retard angiogenesis in the tumors of morphine and naloxone-treated mice $(D)$ with respect to mice treated with morphine alone $(B)$.

naloxone (14), differently from morphine (10), was not able to impair the angiogenesis in tumors of TNBC mice. In order to confirm these results in mice treated with the two substances, micro-vessel formation was examined in breast tumors by injecting FITC dextran into the tail vein of mice. Our results showed that micro-vessel formation was more evident in mice treated with two substances than the controls and mice treated with naloxone alone, but less evident in respect to morphine-treated mice. This result suggests that naloxone is not able to counteract the promoting effect of morphine on tumor angiogenesis (Figure 2A-D). These data were also confirmed by an IHC analysis with CD-31 and measurement of MVD (Figure 3A-E).

Taken together, these results strongly indicate that naloxone counteracts the promoting effects induced by morphine on breast tumor growth, without affecting angiogenesis.

\section{Discussion}

It has been demonstrated that morphine may exert opposite effects on breast cancer growth depending mainly on its concentration (1-11). Several studies reported that morphine induced apoptosis in different Human Tumor Cell Lines (15-17). In our experience (10) and according to the data published by Gupta et al. (9), morphine inhibited apoptosis, promoted proliferation and micro-vessel formation in a breast cancer mouse model, at higher doses. On the contrary, we have recently provided evidence that naloxone, inhibited in vitro and in vivo breast cancer growth without affecting angiogenesis, by acting on a mechanism mediated by p53 (14). In this subsequent investigation, we aimed to dissect the effects of naloxone and morphine in an animal model of human breast cancer. Our results demonstrated that naloxone was able to counteract the promoting effects of morphine on tumor growth. No impairment of micro-vessel formation in tumors of treated mice was observed. Further studies are ongoing in our laboratory in order to elucidate the molecular mechanisms underlying the effects of the combination of morphine and naloxone in the initiation and in the progression of breast cancer. Data emerging from these studies will allow us to clarify the molecular mechanism underlying the effects of morphine and naloxone in the regulation of triple negative breast cancer. 

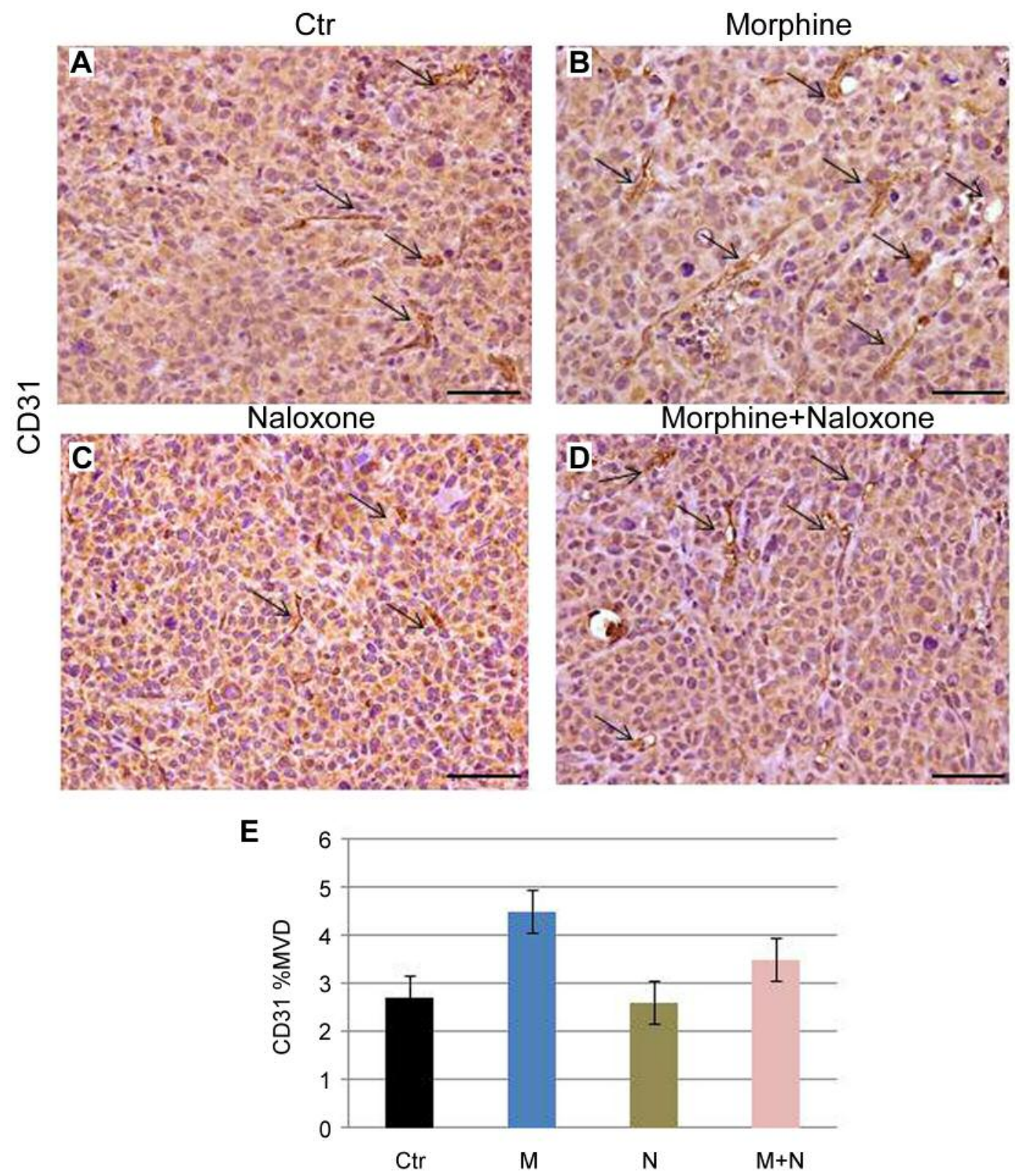

Figure 3. The effects of morphine and naloxone on MVD of breast tumors. (A, B) The analysis for CD31, showed an increased effect on CD31 expression (positive cells stained in brown and indicated by arrows) in morphine plus naloxone-treated mice (D), compared to control mice (A), naloxone-treated mice $(C)$, but a reduced expression with respect to mice treated with morphine alone, indicating that naloxone does not counteract the enhancement of micro-vessel formation induced by morphine. Magnifications 40x. Scale bar 140 um. (E) Quantification of IHC assay was represented as a percentage of CD31-positive cells $(M V D)$. Data shown are averages with SD (error bars) from eight individual mice in each group $(p<0.05$, versus controls).

\section{Acknowledgements}

The Authors are grateful to Dr. Alessandra Trocino, Mrs. Maria Cristina Romano, from the National Cancer Institute of Naples for providing excellent bibliographic service and assistance. The Authors are also grateful to Dr. Antonio Luciano from the National
Cancer Institute of Naples for providing technical assistance in experiments with animals, to Dr. Gennaro Russo and Dr. Gennaro Esposito for providing assistance in the formulations of the drug and to Dr. Giosuè Scognamiglio from the National Cancer Institute of Naples, for performing histopathological experiments. Funding: No funds were received in support of this study. 


\section{Conflicts of Interest}

The Authors declare that they have no competing financial interests.

\section{Authors' Contributions}

The present article was mainly written by SB, AB and MC. All Authors contributed toward data analysis, drafting and critically revised the paper, gave final approval of the version to be published, and agreed to be accountable for all aspects of the work.

\section{References}

1 Lickiss JN: Approaching cancer pain relief. Eur J Pain 5: 5-14, 2001. PMID: 11798211. DOI: 10.1053/eujp.2001.0273

2 Cuomo A, Bimonte S, Forte CA, Botti G and Cascella M: Multimodal approaches and tailored therapies for pain management: the trolley analgesic model. J Pain Res 12: 711714, 2019. PMID: 30863143. DOI: 10.2147/JPR.S178910

3 Pasternak GW: Pharmacological mechanisms of opioid analgesics. Clin Neuropharmacol 16: 1-18, 1993. PMID: 8093680. DOI: 10.1097/00002826-199302000-00001

4 Mantyh PW: Cancer pain and its impact on diagnosis, survival and quality of life. Nat Rev Neurosci 7: 797-809, 2006. PMID: 16988655. DOI: $10.1038 / \mathrm{nrn} 1914$

5 Chen Y, Qin Y, Li L, Chen J, Zhang X and Xie Y: Morphine can inhibit the growth of breast cancer MCF-7 cells by arresting the cell cycle and inducing apoptosis. Biol Pharm Bull 40: 16861692, 2017. PMID: 2874004. DOI: 10.1248/bpb.b17-00215

6 Hatzoglou A, Bakogeorgou E and Castanas E: The antiproliferative effect of opioid receptor agonists on the T47D human breast cancer cell line, is partially mediated through opioid receptors. Eur J Pharmacol 296: 199-207, 1996. PMID: 8838457. DOI: 10.1016/0014-2999(95)00703-2

7 Xie N, Khabbazi S, Nassar ZD, Gregory K, Vithanage T, AnandApte B, Cabot PJ, Sturgess D, Shaw PN and Parat MO: Morphine alters the circulating proteolytic profile in mice: functional consequences on cellular migration and invasion. FASEB J 31: 5208-5216, 2017. PMID: 28784632. DOI: 10.1096/fj.201700546R

8 Nguyen J, Luk K, Vang D, Soto W, Vincent L, Robiner S, Saavedra R, Li Y, Gupta P and Gupta K: Morphine stimulates cancer progression and mast cell activation and impairs survival in transgenic mice with breast cancer. Br J Anaesth 113: i4-i13, 2014. PMID: 24861561. DOI: 10.1093/bja/aeu090
9 Gupta K, Kshirsagar S, Chang L, Schwartz R, Law PY, Yee D and Hebbel RP: Morphine stimulates angiogenesis by activating proangiogenic and survival-promoting signaling and promotes breast tumor growth. Cancer Res 62: 4491-4498, 2002. PMID: 12154060 .

10 Bimonte S, Barbieri A, Rea D, Palma G, Luciano A, Cuomo A, Arra $\mathrm{C}$ and Izzo F: Morphine promotes tumor angiogenesis and increases breast cancer progression. Biomed Res Int 2015: 161508, 2015. PMID: 26064880. DOI: 10.1155/2015/161508

11 Bimonte S, Barbieri A, Palma G and Arra C: The role of morphine in animal models of human cancer: does morphine promote or inhibit the tumor growth? Biomed Res Int 2013: 258141, 2013. PMID: 24069592. DOI: 10.1155/2013/258141

12 Kikuchi Y, Kita T, Miyauchi M, Kizawa I, Oomori K and Kato $\mathrm{K}$ : Effects of naloxone on human ovarian cancer cell growth in vitro and in vivo. Jpn J Cancer Res 78: 519-525, 1987. PMID: 3112065.

13 Farooqui M, Geng ZH, Stephenson EJ, Zaveri N, Yee D and Gupta K: Naloxone acts as an antagonist of estrogen receptor activity in MCF-7 cells. Mol Cancer Ther 5: 611-620, 2006. PMID: 16546975. DOI: 10.1158/1535-7163.MCT-05-0016

14 Bimonte S, Barbieri A, Cascella M, Rea D, Palma G, Del Vecchio V, Forte CA, Del Prato F, Arra C and Cuomo A: The effects of naloxone on human breast cancer progression: in vitro and in vivo studies on MDA-MB-231 cells. Onco Targets Ther 11: 185-191, 2018. PMID: 29379300. DOI: 10.2147/OTT.S145780

15 Hatsukari I, Hitosugi N, Ohno R, Hashimoto K, Nakamura S, Satoh K, Nagasaka H, Matsumoto I and Sakagami H: Induction of apoptosis by morphine in human tumor cell lines in vitro. Anticancer Res 27: 857-864, 2007. PMID: 17465212.

16 Hatsukari I, Hitosugi N, Matsumoto I, Nagasaka H and Sakagami H: Induction of early apoptosis marker by morphine in human lung and breast carcinoma cell lines. Anticancer Res 23: 2413-2416, 2003. PMID: 12894522.

17 Kawase M, Sakagami H, Furuya K, Kikuchi H, Nishikawa H, Motohashi N, Morimoto Y, Varga A and Molnar J: Cell deathinducing activity of opiates in human oral tumor cell lines. Anticancer Res 22: 211-214, 2002. PMID: 12017290.

Received March 12, 2019

Revised April 8, 2019

Accepted April 11, 2019 